Research Article

\title{
Dynamic Optimization of Government Data Transmission Based on Blockchain Technology
}

\author{
Sheng Peng, ${ }^{1,2}$ Linkai Zhu $\mathbb{D}^{2,3}$ Zhiming Cai, $^{2}$ Wenjian Liu, ${ }^{2}$ Chunjiang He, ${ }^{4}$ \\ and Weikang Tang ${ }^{1}$ \\ ${ }^{1}$ Zhuhai Yingying Technology Co.,Ltd, Guangzhou, China \\ ${ }^{2}$ Institute of Data Science, City University of Macau, Macau, China \\ ${ }^{3}$ Trusted Computing and Information Assurance Laboratory Institute of Software Chinese Academy of Sciences, Beijing, China \\ ${ }^{4}$ Zhuhai Da Hengqin Science and Technology Development Co.,Ltd, Zhuhai, China
}

Correspondence should be addressed to Linkai Zhu; d19092105151@cityu.mo

Received 27 April 2021; Revised 6 June 2021; Accepted 14 June 2021; Published 6 July 2021

Academic Editor: Fazlullah Khan

Copyright (C) 2021 Sheng Peng et al. This is an open access article distributed under the Creative Commons Attribution License, which permits unrestricted use, distribution, and reproduction in any medium, provided the original work is properly cited.

With the development of terminal technology and the expansion of application fields, the Internet of Things' application value and service requirements continue to increase. Efficient data transmission is a reliable guarantee for the development and application of the Internet of Things. Blockchain technology provides a solution for storing and delivering distributed data. On this basis, taking the Industrial Internet of Things as the research object, a blockchain-based data transmission optimization method was established. First, an undirected complete graph model is used to describe the network scene. A matrix grid model is used to replace the randomly distributed set of data nodes. Then, a double optimization method is proposed. We designed the mathematical description and modeling method of the lattice matching decision problem and designed the artificial neural network to find the optimal solution to the problem. Finally, an example is used to verify the government data transmission method's technical performance and packet loss rate. It has achieved at least $20 \%$ and $30 \%$ improvements in optimizing the network life of static aggregation nodes and data transmission, respectively. While improving the robustness of the network, it also shows a stable advantage in terms of network energy efficiency indicators.

\section{Introduction}

The Internet of Things (IoT) and its terminal perception devices will significantly impact the development of human productivity. The National Intelligence Council has predicted IoT nodes will connect everything in our lives by 2025 , such as food, parcels, furniture, and paper documents $[1,2]$. It can be seen that IoT devices have strong penetration characteristics that can play an active role in daily life. It has a great possibility of becoming an economic growth point and provides real and productive technical power for the global economic recovery [3]. From a technical perspective, the role and impact of the IoT can be understood through radio frequency identification (RFID) and sensor technology. According to certain standards, the integrated positioning system and laser, infrared, and other sensing technologies are based on certain protocols through maturity or development [4]. The network architecture connects things closely related to human beings, shares the data and information therein, and finally realizes intelligent identification and management of everything in the world [5].

The essence of IoT is to digitally collect useful information in the world's entities and their environments, reliably transmit it through various heterogeneous networks, and finally use it in every aspect of life, scientific research, production, etc., to provide data support and decisionmaking systems [6]. The IoT technology has matured in recent years and has been applied in agricultural production, transportation, industrial control, smart grid, smart home, public safety, and many other fields. It can be seen that the Internet of Things represents an Internet information world that connects real things [7]. 
In recent years, with the effective development of related technologies such as sensors, RFID tags, and the Internet of Things, the demand for wearable devices and other application fields has increased. The IoT has become one of today's academic research fields and industrial manufacturing fields. It is a popular technology and will occupy an important position in the future new Internet world.

The Internet of Things uses various sensing devices to connect human exploration and people and things at any time and any place in the range of activities through any network or service to provide massive cross-industry data services. Today, the Internet of Things has been widely used in transportation, health, and public utilities $[8,9]$. In the huge and diverse network environment of the Internet of Things, the reliability of data aggregation has become the basis of the information services of the Internet of Things. The optimization of data transmission has an important role in promoting the data aggregation of the Internet of Things. This paper takes the Industrial Internet of Things as the research object. It establishes a data transmission optimization method based on the blockchain.

The rest of the paper is organized as follows. In Section 2, related work is studied followed by data transmission optimization in Section 3. The artificial neural network is given in Section 4, and verification of use cases is given in Section 5. Finally, Section 6 concludes the paper.

\section{Related Work}

In the following sections, detailed literature is studied in the context of the Internet of Things and blockchain technologies.

2.1. Internet of Things Technology. When the electronic tag passes through this area, it will be exciting. The energy formed by the induced current transmits the information stored in the electronic tag and transmits the information to read and write device to store information in a database or other applications for processing [10]. The schematic diagram of typical IoT structure technology is shown in Figure 1.

The description language for specific entity information is PML, and the services described by PML constitute EPC IS. Hence, ONS needs to provide automated network data services $[11,12]$. The basic architecture of low-power IoT data transmission is shown in Figure 2. The block structure designed in this paper uses hash pointers to ensure the integrity of the stored data and is difficult to tamper with. In addition to maintaining a pointer to the previous block unit, the hash pointer forms a chain structure. At the same time, the hash pointer data structure also maintains the hash value of the previous block data through the hash function. In this way, the chain structure of the blockchain ensures that the stored IoT public key data are difficult to tamper.

2.2. Blockchain Technology. Blockchain technology uses a chain structure to record the entire transaction information and cannot be tampered with, which has a strong role in increasing trust [13]. This technology has a natural match for the transaction-based financial characteristics of the supply chain. Since the introduction of blockchain technology, the application of blockchain technology to the supply chain has accounted for the highest proportion of various financial transaction studies. Related research mainly believes that the blockchain will expand the coverage of the supply chain, reduce the financing burden of SMEs, and promote the securitization of financial assets in the supply chain.

\section{Data Transmission Optimization}

The latest research shows that mobile aggregation nodes can effectively improve the energy efficiency performance of IoT data aggregation. However, environmental or human factors may affect the sink node, and the movement trajectory is restricted. Such mobile convergence nodes are called trajectory-limited mobile convergence nodes [14, 15]. The influence of trajectory-limited mobile convergence nodes on energy efficiency has a high research value. The data transmission optimization problem of rail-type mobile aggregation is studied. The literature researches the optimization problem of highway-type mobile aggregation data transmission. In mountainous areas, rivers, tunnels, smart grid inspection scenarios, or in data aggregation application scenarios restricted by flight restriction and no-fly conditions, the aggregation node may be affected by the natural environment or human factors. They may also move along a fixed trajectory and aggregate data [16].

In the scenario where the trajectory of the mobile convergence node is limited, the energy consumption of the node has a hierarchical characteristic and forms multiple hot spots. Among them, the data nodes around the trajectory have higher requirements for data transmission optimization because they need to relay the information of all data nodes. The randomness of deployment causes uneven energy consumption of all data nodes, forming scattered hot spots. Therefore, in scenarios where the trajectory of mobile convergence nodes is limited, the joint energy efficiency of the nodes needs to be optimized from the above two levels [17]. This section studies the impact of trajectory-constrained mobile aggregation nodes on the energy efficiency of the network. It establishes an energy consumption model through graph theory analysis and refines and models dual data transmission optimization problems. The proposed design is based on an artificial neural network to search for the optimal problem solution. The solution is achieved using a route discovery algorithm based on the grid model shortest path for data reporting.

3.1. Basic Network Model. This section mainly models and analyzes the data aggregation network and further describes and analyzes the network model through the basic idea of graph theory. On this basis, the network node energy consumption is modeled and analyzed [17].

This figure is used to represent the data aggregation scenario of the IoT. In this figure, $n$ static data nodes are randomly deployed. The motion trajectory of the mobile 


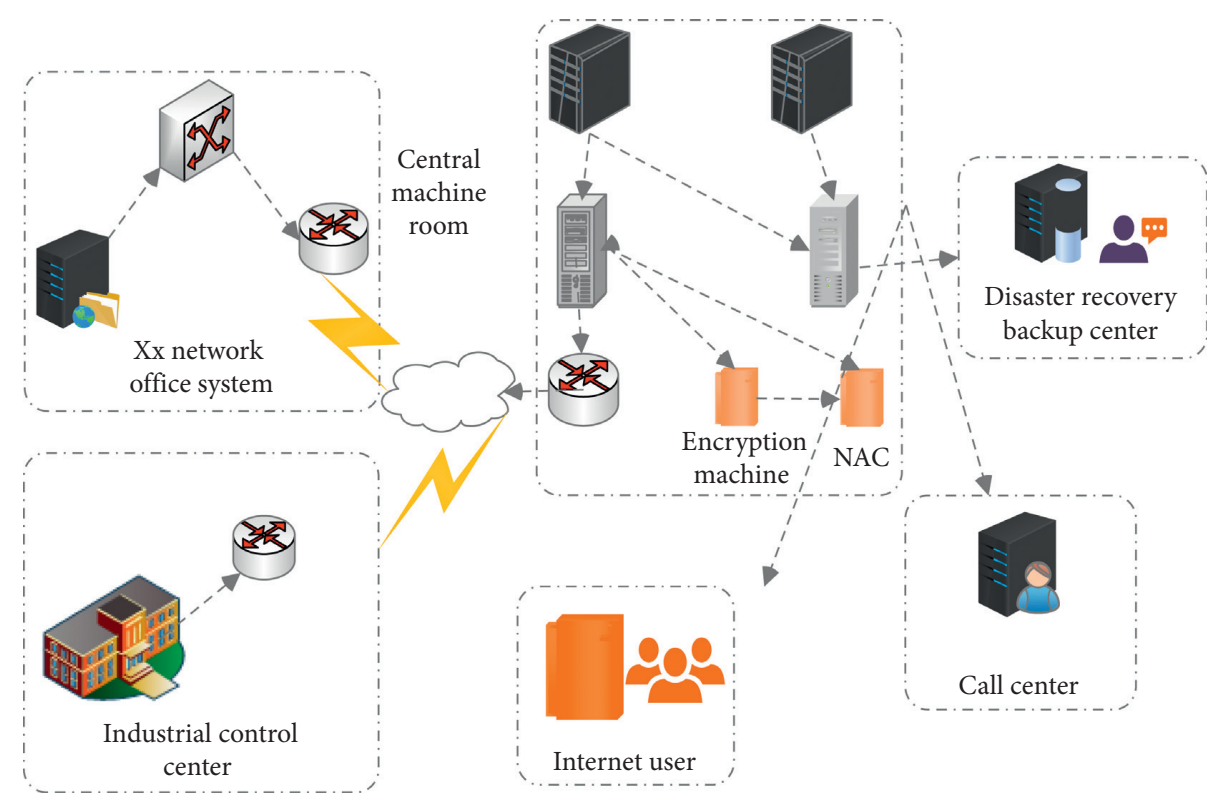

FIGURE 1: Schematic diagram of typical IoT structure technology.

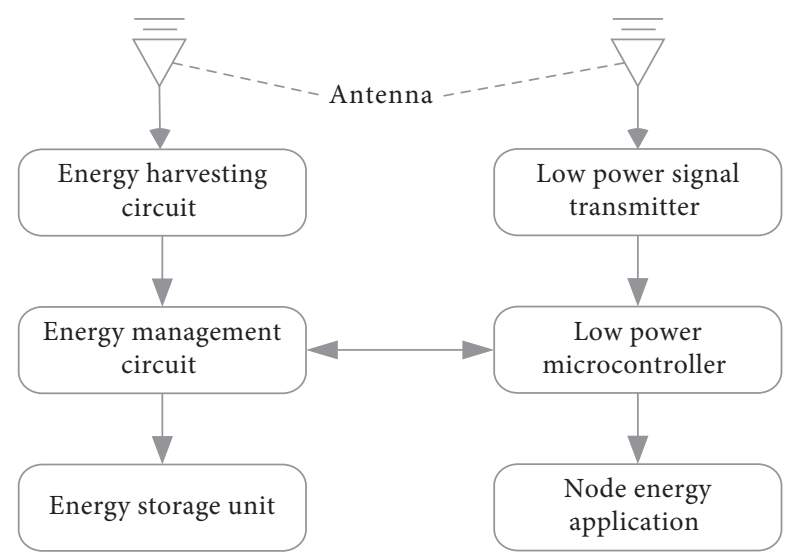

FIgURE 2: Schematic diagram of the basic architecture of low-power IoT data transmission.

convergence node is pm [18]. Na nodes that can perform single-hop communication with Sm nodes on both sides of pm are also called static sink nodes. The schematic diagram of the IoT network model in the scenario of a mobile aggregation node is shown in Figure 3.

In addition, the initial energy of the $\mathrm{Na}$ and $\mathrm{S}$ nodes is E. The $S$ node has sufficient energy and computing power, and it moves back and forth at a constant speed at a rate of $\mathrm{V}$ along with $\mathrm{pm}[18]$. When the $s$ node reaches an endpoint of $\mathrm{pm}$, the $s$ node immediately turns around and travels to the other endpoint of $\mathrm{pm}$ at the same rate. The maximum communication distance of all nodes, including $s$ nodes, is given in $[18,19]$. Each node has sufficient storage space to store routing information and cache information data. In addition, all nodes can obtain and save their location information when the deployment is complete. Since all nodes are stationary, the location information is obtained only once the deployment is completed. The energy consumed by the positioning is negligible.

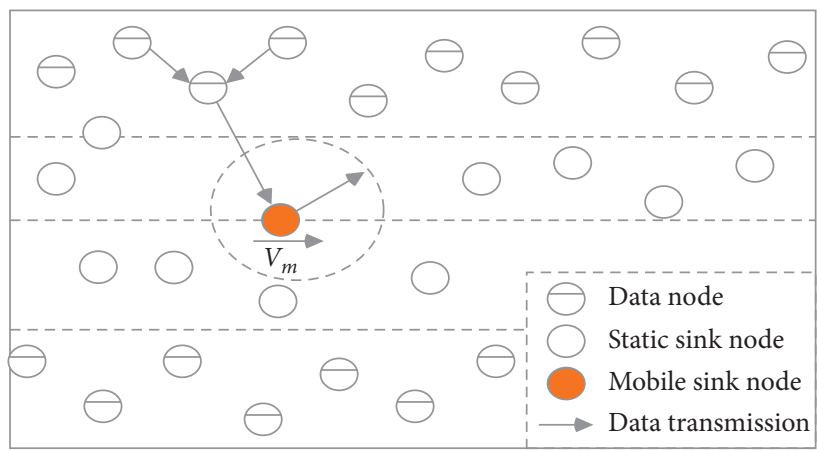

FIGURE 3: Schematic diagram of the IoT network model in the scenario of a mobile data aggregation.

3.2. Description and Analysis of Grid Network Model. Research shows that graph theory can facilitate network model analysis. From the perspective of graph theory, the topology of the Internet of Things or WSN has a high 
similarity with the relationship between the vertices in the basic concept of graph theory. The network nodes can be regarded as the vertex set in the graph object through the reasonable graphical description. The connectivity between the nodes is abstracted as the graph edge set [20]. The data aggregation behavior and network communication behavior through vertices and edges of the graph simplify the network model. It also reduces the computational complexity and further digs into the network model through the relationships between vertices-vertices and vertices-edges. A hierarchical relationship to the connection between nodes is added [21]. The schematic diagram of the IoT network model based on graph theory analysis is shown in Figure 4 [22].

After the graph is divided, Nd nodes with similar position attributes are divided into the same square. The data packets reported by the nodes in the node grid can all reach a specific S node grid through the same path [23]. Therefore, nodes in these same square are considered one entity, converting the described strategy of matching nodes with S nodes into square matching problems. Then, use $g$ to denote the $k^{\text {th }} \mathrm{Na}$ node square matching gas [24]. According to the basic concept of graph theory, the vertices $\mathrm{V}$ and $\mathrm{E}$ constitute G. In this system model, the vertex $v$ is the node in the grid, and the edge $\mathrm{E}$ represents the connectivity between the nodes in the grid. Each pair of different vertices in $G$ has an edge connected and is bidirectional. Hence, $G$ is a complete undirected graph. Through the graph division operation, the computational complexity of the node's matching problem in the network decreases [18].

\section{Artificial Neural Network}

The error backpropagation algorithm is a multilevel feedforward network. It has become the most widely used artificial neural network learning algorithm. It has proposed many improvements to the neural network algorithm [25]. The relationship between nodes and the connectivity between nodes increase the hierarchical relationship. [26]. By adjusting the weight coefficients and thresholds of the input layer and the hidden layer, the hidden layer and the output layer, the training process can reduce the training error along the gradient direction. It forms the threshold set when the error is the smallest and completes the training [27].

4.1. Feed-Forward Calculation. The neural network must train the network before use. Part of the sample data is used for learning and training, and the other part is used for prediction. Assuming that there are $\mathrm{N}$ samples used as learning sample data, the input information and output information of a certain sample are selected for training the network first, and the output information is obtained after training [28]. This process can be expressed with a formula, and the information of the hidden layer can be obtained first:

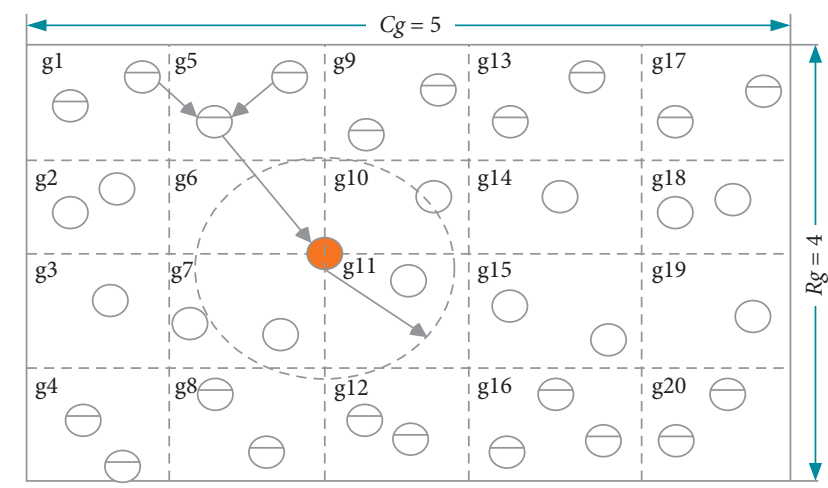

FIGURE 4: Schematic diagram of IoT network model based on graph theory analysis.

$$
\operatorname{net}_{p j}=\text { net }_{j}=\sum_{i=1}^{M} \omega_{i j} o_{i} .
$$

The output of the first node is

$$
\mathrm{o}_{j}=f\left(\text { net }_{j}\right) \text {. }
$$

Use sigmoid function as activation function.

$$
f\left(\text { net }_{j}\right)=\frac{1}{1+\exp \left[-\left(\text { net }_{j}-\theta_{j}\right)\right]} .
$$

The differential function of equation (3) is

$$
f^{\prime}\left(\text { net }_{j}\right)=f\left(\text { net }_{j}\right)\left[1-f\left(\text { net }_{j}\right)\right] .
$$

By adjusting the value of the weighting coefficient of the hidden layer, the information of the first node obtained by the hidden layer is transmitted to the corresponding node of the next layer. The input value of the output layer can be expressed as [29]

$$
\text { net }_{k}=\sum_{j=1}^{q} \omega_{j k} o_{j}
$$

where $q$ is the number of nodes in the hidden layer. Therefore, the actual output data of the first neuron in the output layer are

$$
o_{k}=f\left(\text { net }_{k}\right) .
$$

If there is a difference between the output result of the network training and the target value, the error obtained by the training is passed back from the output layer back. Through a series of corrections, the output information on each node in the weight coefficient is corrected, so that the final output result is infinitely close to the target value [30]. Following the same steps, train $\mathrm{N}$ learning samples to complete the learning process [31]. 
4.2. Weight Adjustment. The error function of the sample can be expressed as

$$
E_{p}=\frac{1}{2} \sum_{k=1}^{L}\left(d_{p k}-o_{p k}\right)^{2} .
$$

The system's average error function is

$$
E_{p}=\frac{1}{2 p} \sum_{p=1}^{N} \sum_{k=1}^{L}\left(d_{p k}-o_{p k}\right)^{2} .
$$

The purpose of neural network learning is to continuously adjust the weighting coefficients by finding the shortest path to reduce the error to reach the optimal value of the weighting coefficients [32].

Equation (8) can be simply expressed as

$$
E=\frac{1}{2} \sum_{k=1}^{L}\left(d_{k}-o_{k}\right)^{2}
$$

4.3. Adjustment of Output Layer Weight Coefficient. In order to make the network output value close to the target value and minimize the error $E$, the weighting coefficient is adjusted according to the gradient algorithm:

$$
\Delta \omega_{j k}=-\eta \frac{\partial \mathrm{E}}{\partial \omega_{j k}},
$$

where $\eta$ is the learning rate.

Therefore, the correction formula for the weighting coefficient of any neuron in the output layer is

$$
\Delta \omega_{j k}=\eta\left(d_{k}-o_{k}\right) f^{\prime}\left(\text { net }_{k}\right) o_{j}=\eta \delta_{k} o_{j} .
$$

\section{Verification of Actual Use Cases for Government Data Transmission}

5.1. Simulation Parameter Setting. In this section, the data transmission optimization method proposed above will be simulated and verified. The host uses an Intel Core i7 $64 \mathrm{~m}$ dual-core processor and $8 \mathrm{~GB}$ running memory. In the simulation program, the wireless transmission standard is $\mathrm{WiFi}$, the physical layer standard is $80211 \mathrm{~b}$, and the MAC type is Ashco. The wireless propagation delay model is the constant speed propagation delay model. The propagation loss model is the range propagation loss model. In the subsequent simulation verification, the same system environment and wireless transmission model are used. In the simulation, this paper's data transmission optimization model will be compared with other methods.

5.2. Data Test. A large amount of lost data is accumulated, and the real-time resuming cannot be continued. Figure 5 shows the test results of the packet loss rate error of
Industrial Internet of Things data transmission. Figure 6 shows the result of the data transmission response error of the Industrial IoT.

Through the performance test of the above indicators, the relevant results are shown in Figures 6 and 7. According to the analysis of the test results, the test standards for related indicators have already met the requirements and experimental settings for dynamic optimization of Industrial Internet of Things data transmission. Next, we will conduct further in-depth tests for other indicators. The above has tested the encryption and decryption of communication data and the blockchainization of stored data of the entire security architecture. The public key data of the IoT communication partner were tested for asymmetric encryption and decryption to achieve the expected results. The generation of the symmetric session key between IoT devices was also tested and finally generated by both IoT communication parties. The session symmetric key is the same for the blockchainization of stored data. It mainly tests the consensus process of the Raft cluster and the query process of blockchain data.

\subsection{Comparison of Government Affairs Data Transmission} Technology. In order to verify the reliability of the parameters, the data transmission optimization technology is used. The test tool used in the test scenario of the result page is a user cycle tool, and related data are generated from this. The following experimental results are obtained, which are shown in Figures 7 and 8. Figure 7 compares the data transmission performance of the Industrial IoT at Standard 1.

Figure 8 shows the comparison result of the data transmission performance of the Industrial IoT at Standard 2.

It can be seen from Figures 7 and 8 that the data transmission performance of the Industrial Internet of Things meets the relevant requirements. This paper has achieved a better performance advantage under the condition of Standard 2. This model is conducive to the relative performance and the efficiency of the actual application process. Figure 8 shows a comparison of the data transmission performance of the Industrial Internet of Things in the case of Standard 2. This comparison also verifies the applicability of the model in this article under different standards. For in-depth exploration in the future, the model established in this article provides a better reference. In general, the above experimental tests meet the security architecture requirements in terms of data communication encryption and data storage reliability. However, the random cycle value setting for Raft cluster heartbeat detection needs to be further improved in future practice. When the performance of the physical device allows, the random cycle value should be reduced as much as possible, which is more in line with the real-time nature of IoT communication. 


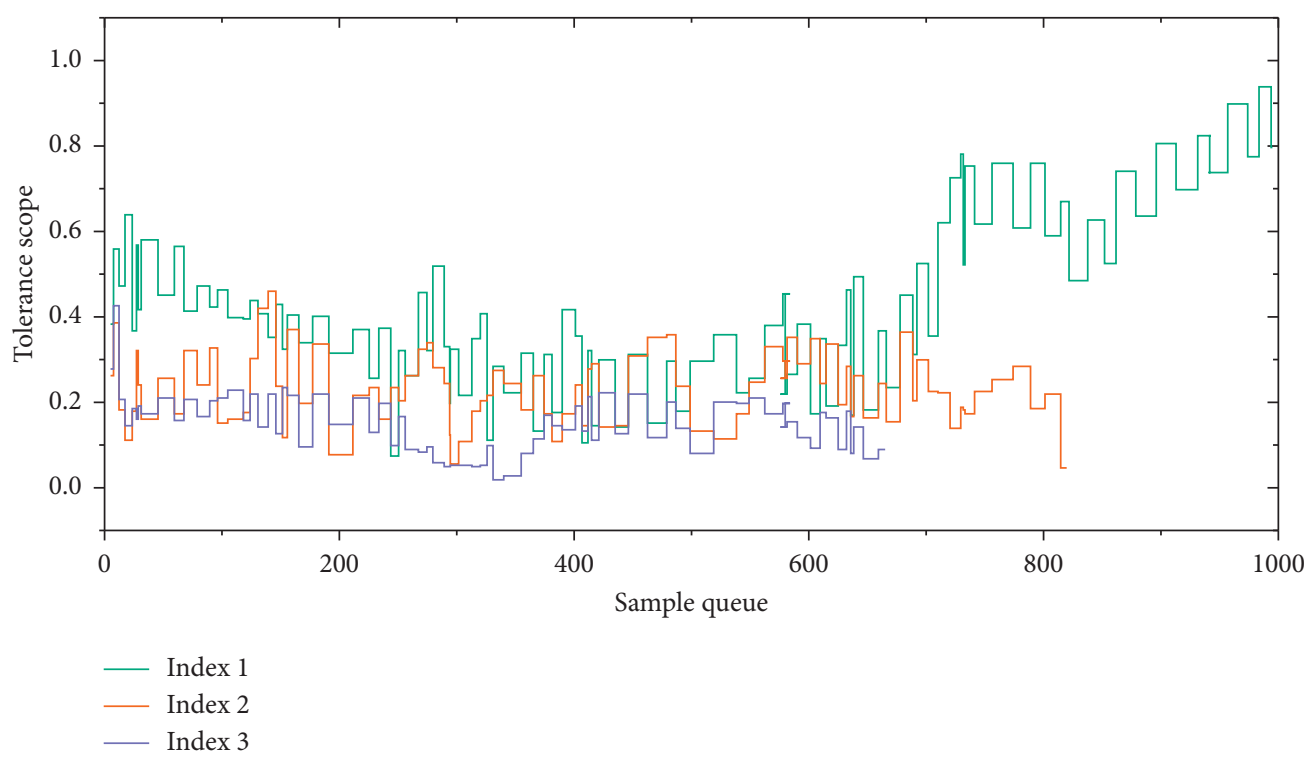

FIgURE 5: Industrial IoT data transmission packet loss rate error test results.

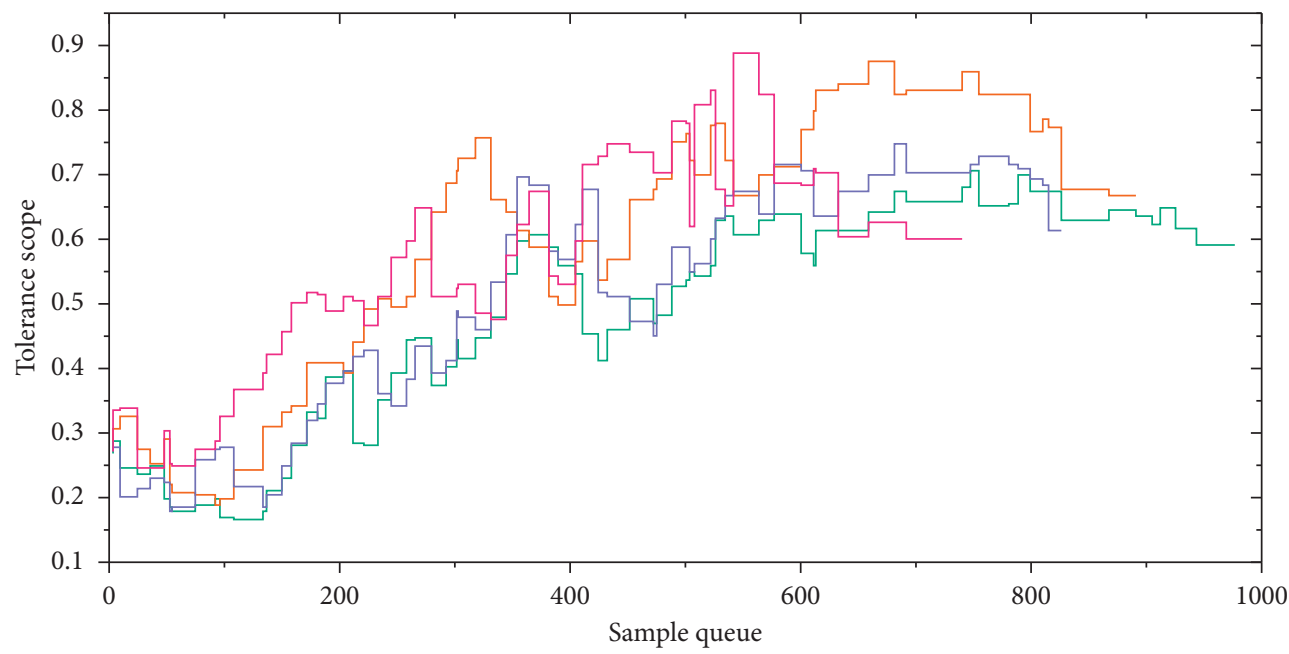

$\begin{array}{ll}\text { Index } 1 & -\operatorname{Index} 3 \\ \text { Index } 2 & -\operatorname{Index} 4\end{array}$

Figure 6: Industrial IoT data transmission response error results.

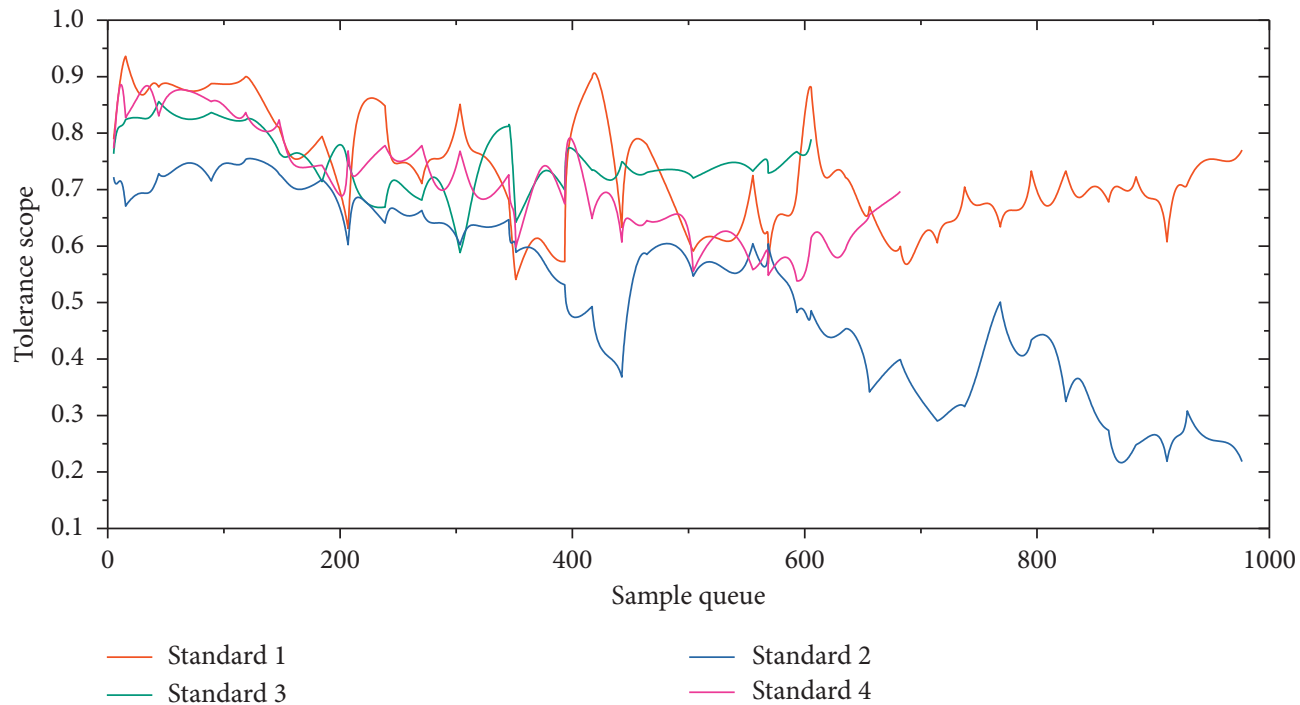

FIgURE 7: Industria Internet of Things data transmission performance comparison: Standard 1. 


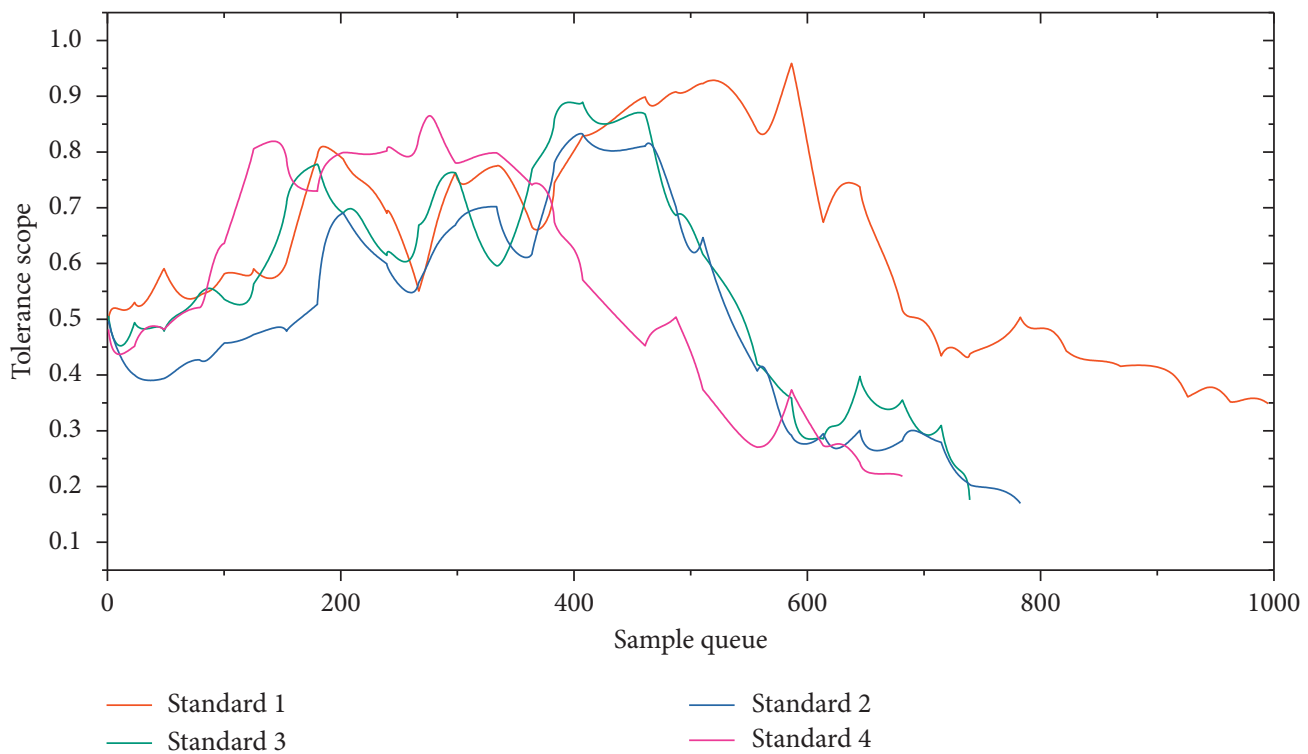

Figure 8: Industrial IoT data transmission performance comparison: Standard 2.

\section{Conclusion}

In the Industrial IoT scenario, the data are aggregated through nodes with limited trajectories. This paper divides the network scene into a grid matrix model with location information characteristics through the idea of graph theory and designs an artificial neural network to seek effective matching. The proposed strategies balance the energy consumption between node grids and reduce the overall energy consumption of network nodes. We have obtained better results using optimization methods of government data transmission with the continuous expansion of the application field of the IoT. With the expansion of new technologies, there are still many problems worthy of research in data transmission optimization. We will further explore optimization methods suitable for government data transmission to provide scientific references for the development of related industries.

\section{Data Availability}

The datasets used and/or analyzed during the current study are available from the corresponding author on reasonable request.

\section{Conflicts of Interest}

The authors declare that they have no conflicts of interest.

\section{Acknowledgments}

This research was supported by the (1) project funded by Zhuhai Industry-University-Research Cooperation Project: Research on Key Technologies of Cross-domain Data Compliance and Mutual Trust Computing in Zhuhai and Macau (No. ZH22017002200011PWC), (2) Research on knowledge-oriented probabilistic graphical model theory based on multi-source data (FDCT-NSFC Projects: 0066/
2019/AFJ), and (3) Research and Application of Cooperative Multi-Agent Platform for Zhuhai-Macao Manufacturing Service (0058/2019/AMJ).

\section{References}

[1] X. Zhu, D. Niu, F. Wang, Y. Guo, and M. Jia, "Operation optimization research of circulating cooling water system based on superstructure and domain knowledge," Chemical Engineering Research and Design, vol. 142, pp. 285-297, 2019.

[2] G.-Y. Zhu, X.-W. Ju, and W.-B. Zhang, "Multi-objective sequence optimization of PCB component assembly with GA based on the discrete frechet distance," International Journal of Production Research, vol. 56, no. 11-12, pp. 4017-4034, 2018.

[3] Y. Zhao, Y. Liu, A. Tian, Y. Yu, and X. Du, "Blockchain based privacy-preserving software updates with proof-of-delivery for Internet of Things," Journal of Parallel and Distributed Computing, vol. 132, pp. 141-149, 2019.

[4] R. Zhang, R. Xue, and L. Liu, "Security and privacy on blockchain," ACM Computing Surveys, vol. 52, no. 3, 2019.

[5] K. Zhang, Y. Zhu, S. Maharjan, and Y. Zhang, "Edge intelligence and blockchain empowered $5 \mathrm{G}$ beyond for the industrial Internet of things," IEEE Network, vol. 33, no. 5, pp. 12-19, 2019.

[6] Y. Yu, Y. Li, J. Tian, and J. Liu, "Blockchain-based solutions to security and privacy issues in the Internet of things," IEEE Wireless Communications, vol. 25, no. 6, pp. 12-18, 2018.

[7] Z. Yin, C. Du, J. Liu, X. Sun, and Y. Zhong, "Research on autodisturbance-rejection control of induction motors based on an ant colony optimization algorithm," IEEE Transactions on Industrial Electronics, vol. 65, no. 4, pp. 3077-3094, 2018.

[8] Z. W. Yan, H. N. Bu, and D. H. Zhang, "Dynamic optimization model of flatness target curve based on hybrid intelligent algorithm," Steel Research International, vol. 89, no. 5, 2016.

[9] Z. Xiong, Y. Zhang, C. L. Nguyen, D. Niyato, and N. Guizani, "The best of both worlds: a general architecture for data management in blockchain-enabled internet-of-things," IEEE Network, vol. 34, 2020. 
[10] Z. Xiong, Y. Zhang, D. Niyato, P. Wang, and Z. Han, "When mobile blockchain meets edge computing," IEEE Communications Magazine, vol. 56, no. 8, pp. 33-39, 2018.

[11] K. Wang, "Research and application of a combined model based on multi-objective optimization for multi-step ahead wind speed forecasting," energy, vol. 60, 2017.

[12] L. Tseng, L. Wong, S. Otoum, M. Aloqaily, and J. B. Othman, "Blockchain for managing heterogeneous Internet of things: a perspective architecture," IEEE Network, vol. 34, no. 1, pp. 16-23, 2020.

[13] M. Shen, H. Liu, L. Zhu et al., "Blockchain-assisted secure device authentication for cross-domain industrial IoT," IEEE Journal on Selected Areas in Communications, vol. 38, no. 5, pp. 942-954, 2020.

[14] J. H. Ryu, P. K. Sharma, J. H. Jo, and J. H. Park, “A blockchainbased decentralized efficient investigation framework for IoT digital forensics," Journal of Supercomputing, vol. 16, 2019.

[15] J. Qiao, C. Duan, H. Jiang, Y. Zhao, and J. Wu, "Research on screening mechanism and parameters optimization of equal thickness screen with variable amplitude based on DEM simulation," Powder Technology, vol. 331, 2018.

[16] X. Ling, Y. Le, J. Wang, and Z. Ding, "Hash access: trustworthy grant-free IoT access enabled by blockchain radio access networks," IEEE Network, vol. 34, no. 1, pp. 54-61, 2020.

[17] T. Li, X. Zhou, H. O. Ikhumhen, and A. Difei, "Research on the optimization of air quality monitoring station layout based on spatial grid statistical analysis method," Environmental Technology, vol. 26, pp. 1-13, 2017.

[18] B. Lee and J. H. Lee, "Blockchain-based secure firmware update for embedded devices in an Internet of Things environment," The Journal of Supercomputing, vol. 73, no. 3, pp. 1152-1167, 2017.

[19] L. Jiang, S. Xie, S. Maharjan, and Y. Zhang, "blockchain empowered wireless power transfer for green and secure Internet of Things," IEEE Network, vol. 33, no. 6, pp. 1-8, 2019.

[20] D. Huang and X. L. Luo, "Process transition based on dynamic optimization with the case of a throughput fluctuating ethylene column," Industrial \& Engineering Chemistry Researc, vol. 57, no. 8, 2018.

[21] Y. Hao, D. Guo, W. Kai, Z. Jiang, Y. Lyu, and X. Ju, "Hyperconnected network: a decentralized trusted computing and networking paradigm," IEEE Network, vol. 32, no. 1, pp. 112-117, 2018.

[22] H. Liang, J. Zou, K. Zuo, and M. J. Khan, "An improved genetic algorithm optimization fuzzy controller applied to the wellhead back pressure control system," Mechanical Systems and Signal Processing, vol. 142, Article ID 106708, 2020.

[23] G. Fortino, F. Messina, D. Rosaci, and G. M. L. Sarne, "Using blockchain in a reputation-based model for grouping agents in the Internet of things," IEEE Transactions on Engineering Management, vol. 14, no. 9, pp. 1-13, 2019.

[24] M. A. Ferrag and L. Maglaras, "DeepCoin: a novel deep learning and blockchain-based energy exchange framework for smart grids," IEEE Transactions on Engineering Management, vol. 63, 2019.

[25] W. Bo and W. Zheng, "Research on real-time optimization decision of energy storage system based on improved Q-learning algorithm," Electrical Engineering, vol. 18, 2018.

[26] J. Qi, G. Jiang, G. Li, Y. Sun, and B. Tao, "Intelligent humancomputer interaction based on surface EMG gesture recognition," IEEE Access, vol. 7, pp. 61378-61387, 2019.

[27] C. Lin, N. Xiong, J. H. Park, and T.-H. Kim, "Dynamic power management in new architecture of wireless sensor networks," International Journal of Communication Systems, vol. 22, no. 6, pp. 671-693, 2009.

[28] F. Long, N. Xiong, A. V. Vasilakos, L. T. Yang, and F. Sun, "A sustainable heuristic QoS routing algorithm for pervasive multi-layered satellite wireless networks," Wireless Networks, vol. 16, no. 6, pp. 1657-1673, 2010.

[29] J. Li, N. Xiong, J. H. Park, C. Liu, S. Ma, and S. Cho, "Intelligent model design of cluster supply chain with horizontal cooperation," Journal of Intelligent Manufacturing, vol. 23, no. 4, pp. 917-931, 2012.

[30] W. Guo, N. Xiong, A. V. Vasilakos, G. Chen, and C. Yu, "Distributed k-connected fault-tolerant topology control algorithms with PSO in future autonomic sensor systems," International Journal of Sensor Networks, vol. 12, no. 1, pp. 53-62, 2012.

[31] H. Liang, J. Zou, Z. Li, M. J. Khan, and Y. Lu, "Dynamic evaluation of drilling leakage risk based on fuzzy theory and PSO-SVR algorithm," Future Generation Computer Systems, vol. 95, pp. 454-466, 2019.

[32] Y. Liu, M. Ma, X. Liu, N. Xiong, A. Liu, and Y. Zhu, "Design and analysis of probing route to defense sink-hole attacks for Internet of Things security," IEEE Transactions on Network Science and Engineering, vol. 7, no. 1, pp. 356-372, 2018. 